International Journal of Pure and Applied Mathematics

Volume 103 No. 4 2015, 675-682

ISSN: 1311-8080 (printed version); ISSN: 1314-3395 (on-line version)

url: http://www.ijpam.eu

doi: http://dx.doi.org/10.12732/ijpam.v103i4.7

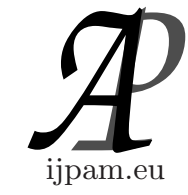

\title{
NEW INTEGRAL TRANSFORM FOR SOLVING SOME FRACTIONAL DIFFERENTIAL EQUATIONS
}

\author{
A. Kashuri ${ }^{1}$ §, A. Fundo ${ }^{2}$, R. Liko ${ }^{3}$ \\ ${ }^{1,3}$ Department of Mathematics \\ University Ismail Qemali \\ Vlora, 9400, ALBANIA \\ ${ }^{2}$ Department of Mathematics \\ Polytechnic University \\ Tirana, 1000, ALBANIA
}

\begin{abstract}
In this paper a new integral transform was applied to solve some families of fractional differential equations. The methodology presented here is based chiefly upon some general theorems on (explicit) particular solutions of some families of fractional differential equations with the new integral transform and the expansion coefficients of binomial series.
\end{abstract}

\section{AMS Subject Classification: 34A08}

Key Words: new integral transform, fractional-order differential equations, Riemann-Liouville fractional integrals, gamma function, Mittag-Leffler function, Wright function

\section{Introduction}

A new integral transform is derived from the classical Fourier integral. A new integral transform (see [1],[3]) was introduced by PhD student Artion Kashuri and Associate Professor Akli Fundo to facilitate the process of solving fractional differential equations in the time domain. Typically, Fourier, Laplace, Sumudu and Elzaki transforms are the convenient mathematical tools for solving differential equations. A new integral transform is defined for functions

Received: April 25, 2015

(C) 2015 Academic Publications, Ltd.

$\S_{\text {Correspondence author }}$ url: www.acadpubl.eu 
of exponential order. This transform has deeper connection with the Laplace transform. The purpose of this study is to show the applicability of this interesting new transform and its efficiency in solving fractional differential equations. New integral transform is a powerful tool and fullness of these lies in their ability to transform differential equations into algebraic equations which allows simple and systematic solution procedures. In the past two decades, the widely investigated subject of fractional calculus has remarkably gained importance and popularity due to its demonstrated applications in numerous diverse fields of science and engineering. These contributions to the fields of science and engineering are based on the mathematical analysis. It covers the widely known classical fields such as Abel's integral equation and viscoelasticity (see [2]). In this paper, we apply the new integral transform of the fractional derivative and the expansion coefficients of binomial series to derive the explicit solutions to homogeneous fractional differential equations. We present some useful definitions and preliminaries as follows.

Definition 1. The fractional derivative of a causal function $f(t)$ is defined by

$$
\frac{d^{\alpha} f(t)}{d t^{\alpha}}= \begin{cases}f^{(n)}(t), & \alpha=n \in N, \\ \frac{1}{\Gamma(n-\alpha)} \int_{0}^{t} \frac{f^{(n)}(t)}{(t-x)^{\alpha-n+1}} d t, & n-1<\alpha<n .\end{cases}
$$

where the Euler gamma function $\Gamma($.$) is defined by$

$$
\Gamma(z)=\int_{0}^{+\infty} t^{z-1} e^{-t} d t \quad(\operatorname{Re}(z)>0)
$$

Definition 2. The new integral transform of a function $f(t)$, $t \in(0,+\infty)$ is defined by

$$
\mathcal{K}[f(t)](v)=A(v)=\frac{1}{v} \int_{0}^{+\infty} e^{-\frac{\mathrm{t}}{v^{2}}} f(t) d t \quad(v \in C)
$$

Definition 3. The Mittag-Leffler function is defined by

$$
E_{\alpha, \beta}(z)=\sum_{k=0}^{+\infty} \frac{z^{k}}{\Gamma(\alpha k+\beta)} \quad(z, \alpha, \beta \in C, \operatorname{Re}(\alpha)>0)
$$

Definition 4. The simplest Wright function is defined by

$$
\phi(\alpha, \beta ; z)=\sum_{k=0}^{+\infty} \frac{1}{\Gamma(\alpha k+\beta)} \cdot \frac{z^{k}}{k !} \quad(z, \alpha, \beta \in C)
$$


Definition 5. The general Wright function ${ }_{p} \Psi_{q}(z)$ is defined for $z \in C$, complex $a_{i}, b_{j} \in C$, and real $\alpha_{i}, \beta_{j} \in R$ $(i=1, \ldots, p ; j=1, \ldots, q)$ by the series

$$
{ }_{p} \Psi_{q}(z)={ }_{p} \Psi_{q}\left[\begin{array}{c}
\left(a_{i}, \alpha_{i}\right)_{1, p} \\
\left(b_{j}, \beta_{j}\right)_{1, q}
\end{array} \mid z\right]=\sum_{k=0}^{+\infty} \frac{\prod_{i=1}^{p} \Gamma\left(a_{i}+\alpha_{i} k\right)}{\prod_{j=1}^{q} \Gamma\left(b_{j}+\beta_{j} k\right)} \cdot \frac{z^{k}}{k !}
$$

where $z, a_{i}, b_{j} \in C, \alpha_{i}, \beta_{j} \in R, i=1, \ldots, p$ and $j=1, \ldots, q$.

Definition 6. The Riemann-Liouville fractional derivatives $D_{a+}^{\alpha} y$ and $D_{b-}^{\alpha} y$ of order $\alpha \in C,(\operatorname{Re}(\alpha) \geq 0)$ are defined by

$$
\begin{aligned}
&\left(D_{a+}^{\alpha} y\right)(x)=\frac{1}{\Gamma(n-\alpha)}\left(\frac{d}{d x}\right)^{n} \int_{a}^{x} \frac{y(t) d t}{(x-t)^{\alpha-n+1}} \\
& \quad(n=[\operatorname{Re}(\alpha)]+1 ; x>a)
\end{aligned}
$$

and

$$
\begin{aligned}
\left(D_{b-}^{\alpha} y\right)(x)=\frac{1}{\Gamma(n-\alpha)}\left(-\frac{d}{d x}\right)^{n} \int_{x}^{b} \frac{y(t) d t}{(t-x)^{\alpha-n+1}} & (n=[\operatorname{Re}(\alpha)]+1 ; x<b),
\end{aligned}
$$

respectively, where $[\operatorname{Re}(\alpha)]$ means the integral part of $\operatorname{Re}(\alpha)$.

\section{Preliminaries}

1. $\mathcal{K}[\phi(\alpha, \beta ; z)](v)=v E_{\alpha, \beta}\left(v^{2}\right), \quad(\alpha>-1, \beta \in C ; \operatorname{Re}(v)>0)$.

2. The new integral transform of the generalized Wright function is given by

$$
\begin{aligned}
& \mathcal{K}\left\{{ }_{p} \Psi_{q}\left[\begin{array}{c}
\left(a_{i}, \alpha_{i}\right)_{1, p} \\
\left(b_{j}, \beta_{j}\right)_{1, q}
\end{array} \mid-t\right]\right\}(v)=v^{2}{ }_{p+1} \Psi_{q}\left[\begin{array}{c}
(1,1),\left(a_{i}, \alpha_{i}\right)_{1, p} \\
\left(b_{j}, \beta_{j}\right)_{1, q}
\end{array} \mid-v^{2}\right] \\
&(\operatorname{Re}(v)>0), i=1,2, \ldots, p \text { and } j=1,2, \ldots, q . \\
& \text { 3. } \mathcal{K}\left[D^{\alpha} f(t)\right](v)=\frac{\mathcal{K}[f(t)](v)}{v^{2 \alpha}}-\sum_{k=1}^{n} \frac{f^{(k-1)}(0)}{v^{2(\alpha-k)-1}},
\end{aligned}
$$

where $\alpha>0, n-1<\alpha \leq n \in N, f(t) \in C^{n}(0,+\infty) f^{(n)}(t) \in L_{1}(0, b)$ for any $b>0$ (see [4]). The interchange of the order of integration in the above derivation can be justified by applying Fubini's theorem. 


\section{Solutions of the Fractional Differential Equations}

Throughout this section, we let $y(t)$ be such that for some value of the parameter $v$, the new integral transform $\mathcal{K}[f(t)](v)$ converges.

Theorem 7. Let $1<\alpha<2$ and $a, b \in R$. Then the fractional differential equation

$$
y^{\prime \prime}(t)+a y^{(\alpha)}(t)+b y(t)=0
$$

with the initial conditions $y(0)=c_{0}$ and $y^{\prime}(0)=c_{1}$ has its solution given by

$$
\begin{aligned}
y(t)= & c_{0} \sum_{k=0}^{+\infty} \frac{(-b)^{k} t^{2 k}}{k !} \sum_{r=0}^{+\infty} \frac{\Gamma(r+k+1)\left(-a t^{2-\alpha}\right)^{r}}{\Gamma[(2-\alpha) r+2 k+1] r !} \\
& +c_{1} \sum_{k=0}^{+\infty} \frac{(-b)^{k} t^{2 k+1}}{k !} \sum_{r=0}^{+\infty} \frac{\Gamma(r+k+1)\left(-a t^{2-\alpha}\right)^{r}}{\Gamma[(2-\alpha) r+2 k+2] r !} \\
& +a c_{0} \sum_{k=0}^{+\infty} \frac{(-b)^{k} t^{2 k-\alpha+2}}{k !} \sum_{r=0}^{+\infty} \frac{\Gamma(r+k+1)\left(-a t^{2-\alpha}\right)^{r}}{\Gamma[(2-\alpha) r+2 k-\alpha+3] r !} \\
& +a c_{1} \sum_{k=0}^{+\infty} \frac{(-b)^{k} t^{2 k-\alpha+3}}{k !} \sum_{r=0}^{+\infty} \frac{\Gamma(r+k+1)\left(-a t^{2-\alpha}\right)^{r}}{\Gamma[(2-\alpha) r+2 k-\alpha+4] r !} .
\end{aligned}
$$

Proof. Applying the new integral transform (see Preliminary 3) and taking into account, we have

$$
\begin{aligned}
\left(\frac{\mathcal{K}[y]}{v^{4}}-\frac{y(0)}{v^{3}}-\frac{y^{\prime}(0)}{v}\right)+a\left(\frac{\mathcal{K}[y]}{v^{2 \alpha}}-\frac{y(0)}{v^{2(\alpha-1)-1}}-\frac{y^{\prime}(0)}{v^{2(\alpha-2)-1}}\right) & +b \mathcal{K}[y]=0 .
\end{aligned}
$$

Equation (5) yields

$$
\mathcal{K}[y]=\frac{c_{0} v^{2 \alpha-3}+c_{1} v^{2 \alpha-1}+a c_{0} v^{3}+a c_{1} v^{5}}{b v^{2 \alpha}+v^{2 \alpha-4}+a}
$$

Applying the inverse new integral transform to Equation (6) we derive the solution.

Example 2.1. The fractional differential equation of a generalized viscoelastic free damping oscillation

$$
y^{\prime \prime}(t)+a y^{\left(\frac{5}{3}\right)}(t)+b y(t)=0
$$


with the initial conditions $y(0)=c_{0}$ and $y^{\prime}(0)=c_{1}$ has its solution given by

$$
\begin{aligned}
& y(t)=c_{0} \sum_{k=0}^{+\infty} \frac{(-b)^{k} t^{2 k}}{k !} \sum_{r=0}^{+\infty} \frac{\Gamma(r+k+1)\left(-a t^{\frac{1}{3}}\right)^{r}}{\Gamma\left[\frac{1}{3} r+2 k+1\right] r !} \\
& +c_{1} \sum_{k=0}^{+\infty} \frac{(-b)^{k} t^{2 k+1}}{k !} \sum_{r=0}^{+\infty} \frac{\Gamma(r+k+1)\left(-a t^{\frac{1}{3}}\right)^{r}}{\Gamma\left[\frac{1}{3} r+2 k+2\right] r !} \\
& +a c_{0} \sum_{k=0}^{+\infty} \frac{(-b)^{k} t^{2 k+\frac{1}{3}}}{k !} \sum_{r=0}^{+\infty} \frac{\Gamma(r+k+1)\left(-a t^{\frac{1}{3}}\right)^{r}}{\Gamma\left[\frac{1}{3} r+2 k+\frac{4}{3}\right] r !} \\
& +a c_{1} \sum_{k=0}^{+\infty} \frac{(-b)^{k} t^{2 k+\frac{4}{3}}}{k !} \sum_{r=0}^{+\infty} \frac{\Gamma(r+k+1)\left(-a t^{\frac{1}{3}}\right)^{r}}{\Gamma\left[\frac{1}{2} r+2 k+\frac{7}{3}\right] r !}
\end{aligned}
$$

In particular, if $a=\sqrt{2}$ and $b=5$, then the equation

$$
y^{\prime \prime}(t)+\sqrt{2} y^{\left(\frac{5}{3}\right)}(t)+5 y(t)=0
$$

with the initial conditions $y(0)=c_{0}$ and $y^{\prime}(0)=c_{1}$ has its solution given by

$$
\begin{aligned}
& y(t)=c_{0} \sum_{k=0}^{+\infty} \frac{(-5)^{k} t^{2 k}}{k !} \sum_{r=0}^{+\infty} \frac{\Gamma(r+k+1)\left(-\sqrt{2} t^{\frac{1}{3}}\right)^{r}}{\Gamma\left[\frac{1}{3} r+2 k+1\right] r !} \\
& +c_{1} \sum_{k=0}^{+\infty} \frac{(-5)^{k} t^{2 k+1}}{k !} \sum_{r=0}^{+\infty} \frac{\Gamma(r+k+1)\left(-\sqrt{2} t^{\frac{1}{3}}\right)^{r}}{\Gamma\left[\frac{1}{3} r+2 k+2\right] r !} \\
& +\sqrt{2} c_{0} \sum_{k=0}^{+\infty} \frac{(-5)^{k} t^{2 k+\frac{1}{3}}}{k !} \sum_{r=0}^{+\infty} \frac{\Gamma(r+k+1)\left(-\sqrt{2} t^{\frac{1}{3}}\right)^{r}}{\Gamma\left[\frac{1}{3} r+2 k+\frac{4}{3}\right] r !} \\
& +\sqrt{2} c_{1} \sum_{k=0}^{+\infty} \frac{(-5)^{k} t^{2 k+\frac{4}{3}}}{k !} \sum_{r=0}^{+\infty} \frac{\Gamma(r+k+1)\left(-\sqrt{2} t^{\frac{1}{3}}\right)^{r}}{\Gamma\left[\frac{1}{2} r+2 k+\frac{7}{3}\right] r !}
\end{aligned}
$$

Theorem 8. Let $1<\alpha \leq 2$ and $a, b \in R$. Then the fractional differential equation

$$
y^{(\alpha)}(t)+a y^{\prime}(t)+b y(t)=0
$$


with the initial conditions $y(0)=c_{0}$ and $y^{\prime}(0)=c_{1}$ has its solution given by

$$
\begin{array}{r}
y(t)=c_{0} \sum_{k=0}^{+\infty} \frac{(-b)^{k}}{k !} \sum_{r=0}^{+\infty} \frac{\Gamma(r+k+1)(-a)^{r} t^{(\alpha-1) r+\alpha k}}{\Gamma[(\alpha-1) r+\alpha k+1] r !} \\
+c_{1} \sum_{k=0}^{+\infty} \frac{(-b)^{k}}{k !} \sum_{r=0}^{+\infty} \frac{\Gamma(r+k+1)(-a)^{r} t^{(\alpha-1) r+\alpha k+1}}{\Gamma[(\alpha-1) r+\alpha k+2] r !} \\
+a c_{0} \sum_{k=0}^{+\infty} \frac{(-b)^{k}}{k !} \sum_{r=0}^{+\infty} \frac{\Gamma(r+k+1)(-a)^{r} t^{(\alpha-1) r+\alpha k+\alpha-1}}{\Gamma[(\alpha-1) r+\alpha k+\alpha] r !} .
\end{array}
$$

Proof. Applying the new integral transform (see Preliminary 3) and taking into account, we have

$$
\left(\frac{\mathcal{K}[y]}{v^{2 \alpha}}-\frac{y(0)}{v^{2(\alpha-1)-1}}-\frac{y^{\prime}(0)}{v^{2(\alpha-2)-1}}\right)+a\left(\frac{\mathcal{K}[y]}{v^{2}}-\frac{y(0)}{v}\right)+b \mathcal{K}[y]=0
$$

Equation (13) yields

$$
\mathcal{K}[y]=\frac{a c_{0} v^{2 \alpha-1}+c_{0} v^{3}+c_{1} v^{5}}{b v^{2 \alpha}+a v^{2 \alpha-2}+1}
$$

Applying the inverse new integral transform to Equation (14) we derive the solution.

This solution can be expressed by the Wright function as

$$
\begin{aligned}
y(t)= & c_{0} \sum_{k=0}^{+\infty} \frac{(-b)^{k} t^{\alpha k}}{k !}{ }_{1} \Psi_{1}\left[\begin{array}{c}
(k+1,1) \\
(\alpha k+1, \alpha-1)
\end{array} \mid-a t^{\alpha-1}\right] \\
& +c_{1} \sum_{k=0}^{+\infty} \frac{(-b)^{k} t^{\alpha k+1}}{k !}{ }_{1} \Psi_{1}\left[\begin{array}{c}
(k+1,1) \\
(\alpha k+2, \alpha-1)
\end{array} \mid-a t^{\alpha-1}\right] \\
& +a c_{0} \sum_{k=0}^{+\infty} \frac{(-b)^{k} t^{\alpha k+\alpha-1}}{k !}{ }_{1} \Psi_{1}\left[\begin{array}{c}
(k+1,1) \\
(\alpha k+\alpha, \alpha-1)
\end{array} \mid-a t^{\alpha-1}\right] .
\end{aligned}
$$

Example 2.2. If we let $\alpha=\frac{5}{3}, a=1, b=1$ in Theorem 8 , then the equation

$$
y^{\left(\frac{5}{3}\right)}(t)+y^{\prime}(t)+y(t)=0
$$

with the initial conditions $y(0)=c_{0}$ and $y^{\prime}(0)=c_{1}$ has its solution given by

$$
y(t)=c_{0} \sum_{k=0}^{+\infty} \frac{(-1)^{k}}{k !} \sum_{r=0}^{+\infty} \frac{\Gamma(r+k+1)(-1)^{r} t^{\frac{2}{3} r+\frac{5}{3} k}}{\Gamma\left[\frac{2}{3} r+\frac{5}{3} k+1\right] r !}
$$




$$
\begin{aligned}
& +c_{1} \sum_{k=0}^{+\infty} \frac{(-1)^{k}}{k !} \sum_{r=0}^{+\infty} \frac{\Gamma(r+k+1)(-1)^{r} t^{\frac{2}{3} r+\frac{5}{3} k+1}}{\Gamma\left[\frac{2}{3} r+\frac{5}{3} k+2\right] r !} \\
& +c_{0} \sum_{k=0}^{+\infty} \frac{(-1)^{k}}{k !} \sum_{r=0}^{+\infty} \frac{\Gamma(r+k+1)(-1)^{r} t^{\frac{2}{3}} r+\frac{5}{3} k+\frac{2}{3}}{\Gamma\left[\frac{2}{3} r+\frac{5}{3} k+\frac{5}{3}\right] r !}
\end{aligned}
$$

Theorem 9. Let $0<\alpha \leq 1$ and $b \in R$. Then the fractional differential equation

$$
y^{(\alpha)}(t)-b y(t)=0
$$

with the initial condition $y(0)=c_{0}$ has its solution given by

$$
y(t)=c_{0} \sum_{k=0}^{+\infty} \frac{\left(b t^{\alpha}\right)^{k}}{\Gamma(\alpha k+1)}=c_{0} E_{\alpha, 1}\left(b t^{\alpha}\right)
$$

Proof. Applying the new integral transform to Equation (16), that is

$$
\left(\frac{\mathcal{K}[y]}{v^{2 \alpha}}-\frac{y(0)}{v^{2(\alpha-1)-1}}\right)-b \mathcal{K}[y]=0
$$

we have

$$
\mathcal{K}[y]=\frac{c_{0} v^{3}}{1-b v^{2 \alpha}}
$$

Applying the inverse new integral transform to Equation (18) we derive the solution.

Remark. If $a=0$ in Equation (11), then the equation

$$
y^{(\alpha)}(t)+b y(t)=0, \quad 1<\alpha \leq 2
$$

with the initial conditions $y(0)=c_{0}$ and $y^{\prime}(0)=c_{1}$ has its solution given by

$$
\begin{aligned}
y(t) & =c_{0} \sum_{k=0}^{+\infty} \frac{\left(-b t^{\alpha}\right)^{k}}{\Gamma(\alpha k+1)}+c_{1} t \sum_{k=0}^{+\infty} \frac{\left(-b t^{\alpha}\right)^{k}}{\Gamma(\alpha k+2)} \\
& =c_{0} E_{\alpha, 1}\left(-b t^{\alpha}\right)+c_{1} t E_{\alpha, 2}\left(-b t^{\alpha}\right)
\end{aligned}
$$

Theorem 10. A nearly simple harmonic vibration equation

$$
y^{(\alpha)}(t)+\omega^{2} y(t)=0, \quad 1<\alpha \leq 2
$$

with the initial conditions $y(0)=c_{0}$ and $y^{\prime}(0)=c_{1}$ has its solution given by

$$
y(t)=c_{0} E_{\alpha, 1}\left(-\omega^{2} t^{\alpha}\right)+c_{1} t E_{\alpha, 2}\left(-\omega^{2} t^{\alpha}\right)
$$


Proof. Putting $b=\omega^{2}$ in Equation (19).

In fact, by applying the new integral transform to a linear fractional differential equation with the initial conditions, we can easily derive its solutions as the previous forms in this paper.

\section{Conclusion}

In this paper a new integral transform was applied to solve some families of fractional differential equations. The methodology presented here is based chiefly upon some general theorems on (explicit) particular solutions of some families of fractional differential equations with the new integral transform and the expansion coefficients of binomial series. The connection of a new integral transform with the Laplace transform goes much deeper and we can find other relations of a new integral transform by this connection. A new integral transform is a convenient tool for solving fractional differential equations in the time domain.

\section{References}

[1] A. Kashuri, A. Fundo, A new integral transform, Advances in Theoretical and Applied Mathematics, 8 , No. 1 (2013), 27-43.

[2] S. Lin, C. Lu, Laplace transform for solving some families of fractional differential equations and its applications, Advances in Difference Equations (2013), 1-9.

[3] A. Kashuri, A. Fundo, M. Kreku, Mixture of a new integral transform and homotopy perturbation method for solving nonlinear partial differential equations, Advances in Pure Mathematics, 3, No. 3 (2013), 317-323.

[4] A. Kashuri, A. Fundo, Variational iteration method for fractional calculus by new integral transform, In: Conference: NTAM 2014, 7-8 November Tirana, Albania. 